\title{
Anticoagulation with apixaban in a patient with a left ventricular assist device and gastrointestinal bleeding: A viable alternative to warfarin?
}

Francesco Pollari, MD, Theodor Fischlein, MD, Matthias Fittkau, MD, and Giuseppe Santarpino, MD, Nuremberg, Germany

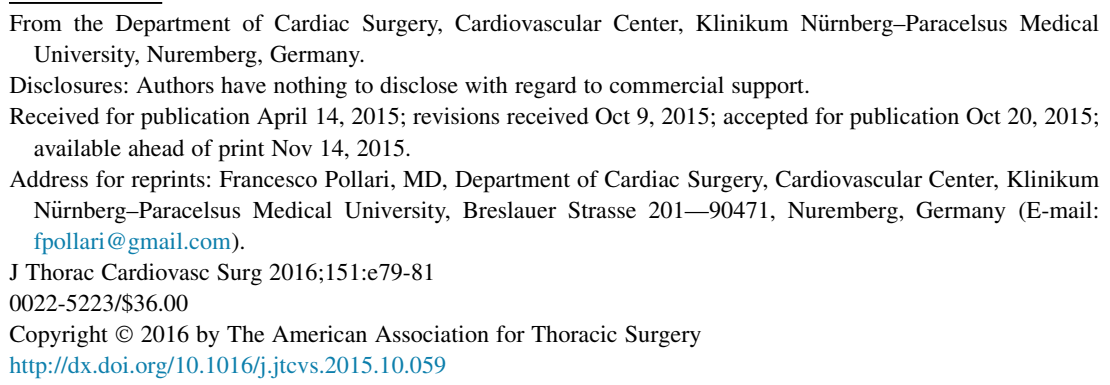

In recent years, left ventricular assist devices (LVADs) have become an acceptable option for patients with end-stage heart failure. LVAD support requires long-term anticoagulation and antiplatelet therapy, however, which carry a high risk of gastrointestinal bleeding. ${ }^{1}$ The most appropriate management of this complication remains a matter of debate. Our aim is to report our experience with apixaban in the management of a patient undergoing LVAD implantation who had multiple gastrointestinal bleeding events.

\section{CLINICAL SUMMARY}

A 78-year-old man was admitted to our center because of refractory ischemic heart failure and an indication for LVAD implantation as destination therapy. Eighteen years previously, he had undergone coronary artery bypass grafting in another institution, and 4 years previously he had undergone a percutaneous coronary intervention with stenting. Five months before the LVAD implantation, the patient had lower gastrointestinal bleeding develop while he was receiving dual antiplatelet therapy (aspirin $100 \mathrm{mg} / \mathrm{d}$ and clopidogrel $75 \mathrm{mg} / \mathrm{d}$ ). This regimen had been established 3 months before the bleeding event as secondary prevention because of an acute coronary syndrome (no invasive procedures were possible). Colonoscopy excluded the presence of angiodysplasia and revealed a polypoid formation (adenoma), which was resected. Result of upper endoscopy were negative. In the absence of active bleeding and other contraindications, HeartMate II (Thoratec Corp, Pleasanton, Calif) implantation was successfully performed (placement of the inflow cannula in the left ventricular apex with the outflow cannula anastomosed to the ascending aorta; Figure 1). At admission, the patient's international normalized ratio (INR) was 1.11 , his creatinine level was $2 \mathrm{mg} / \mathrm{dL}$, and his

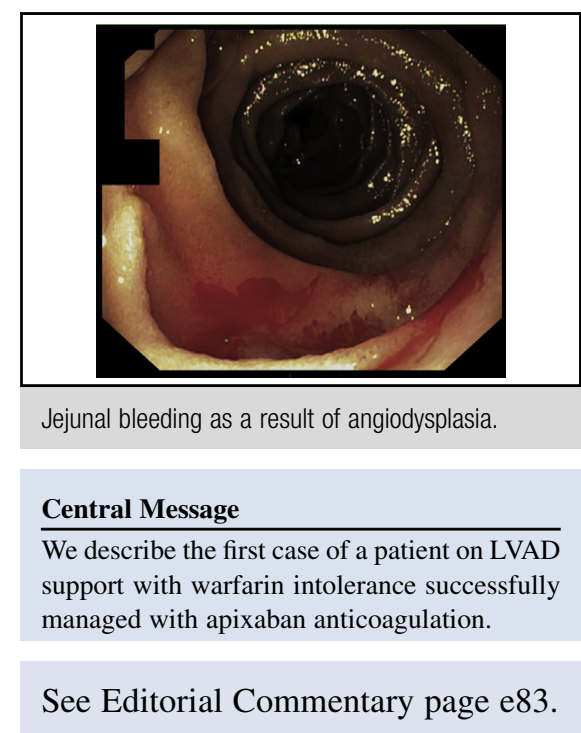

glomerular filtration rate was $31.6 \mathrm{~mL} / \mathrm{min}$. The creatinine and glomerular filtration rate values returned to reference ranges after the postoperation. On postoperative day 22 , the patient had gastrointestinal bleeding develop, with a hemoglobin drop that required blood transfusion. At that time, warfarin had not been already administered, and the patient was receiving unfractionated heparin (partial thromboplastin time, $50 \mathrm{~s}$; INR, 1.23). After comprehensive diagnostic evaluation (hematologic screening test including von Willebrand factor [vWF] activity, 74\%; vWF antigen, $98 \%$, factor VIII, $129 \%$, platelet function analysis, $300 \mathrm{~s}$, abdominal computed tomography, a gastrointestinography, gastroscopy, colonoscopy, and small-bowel capsule endoscopy), multiple angiodysplastic lesions were evident in the upper gastrointestinal tract, and these were treated with argon plasma coagulation. No active bleeding source was seen. Anticoagulation therapy was established with a target INR of 1.5 to 2.0. No aspirin was administered after the first bleeding episode. The patient was discharged 1.8 months postoperatively. During the following 9 months, he was readmitted to the hospital 5 times for repeated episodes of upper and lower gastrointestinal bleeding presenting with either melena or hematochezia. During this period, the INR range was between 1.23 and 2.29. Gastroscopy and colonoscopy always showed new angiodysplastic lesions with active jejunal and colonic bleeding 


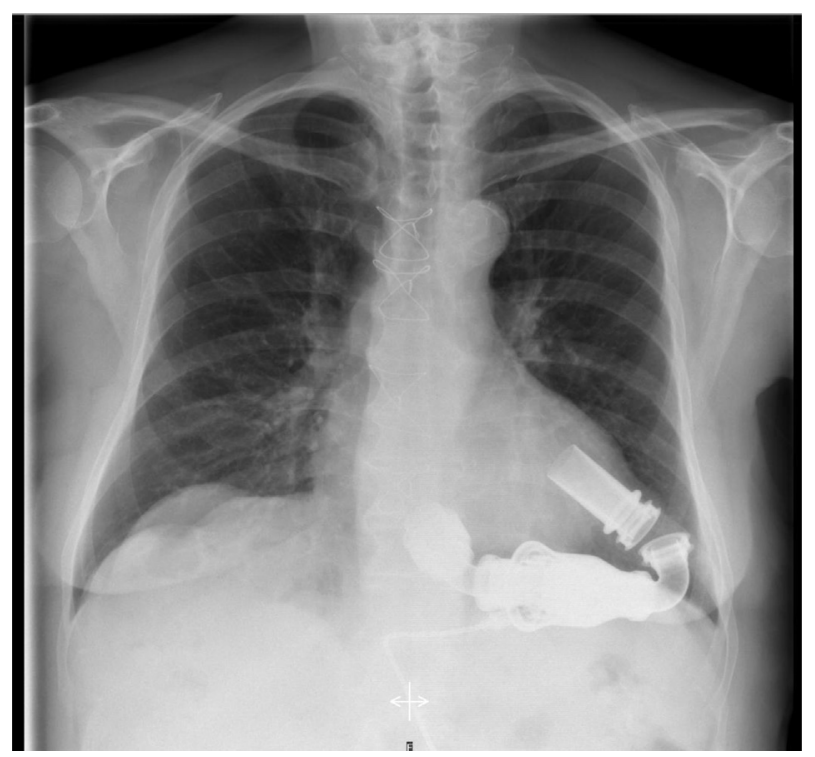

FIGURE 1. Postoperative chest radiograph showing the HeartMate II (Thoratec Corp, Pleasanton, Calif) left ventricular assist device.

(Figure 2), which were treated with either argon plasma coagulation or clipping. A total of 53 blood units were transfused during this period. At the time of each bleeding event, warfarin therapy was discontinued until complete resolution of bleeding, and the patient was given lowmolecular weight heparin. The longest time of warfarin's withholding was 4 weeks. Because of the high risk of new bleeding, after multidisciplinary clinical and ethical discussion that also involved a gastroenterologist, it was decided to start a regimen of apixaban $(2.5 \mathrm{mg}$ twice daily, in accordance with the age-almost 80 years old-and weight $-59 \mathrm{~kg}$ - of the patient). The decision was taken in accordance with the will of the patient and according to the Helsinki Declaration (article 37). After 1 year of apixaban, no episode of gastrointestinal bleeding recurred, with no signs or symptoms of LVAD thrombosis. At follow-up, a coagulation screening showed values of standard tests to be within their reference ranges (Quick test, $82 \%$ [reference value $>70 \%$ ]; INR, 1.13; partial thromboplastin time 31 seconds [reference range, 25-34 seconds]), whereas anti-factor Xa was $191 \mathrm{ng} / \mathrm{mL}$, which might be a sufficient concentration in comparison with the literature. ${ }^{2}$ Other coagulation tests yielded the following results: factor VIII, 89\% (reference range, 60\%-150\%); vWF antigen, 98\% (reference range 50\%-160\%), vWF activity, $54 \%$ (reference range, $46.3 \%-173.2 \%$ ), ratio of vWF activity to antigen, 0.6 (reference range, 0.7-1.3), and anti-platelet factor 4/heparin antibody assay, negative.

\section{DISCUSSION}

Continuous-flow LVADs have been increasingly used during the last decade, resulting in improved outcomes for

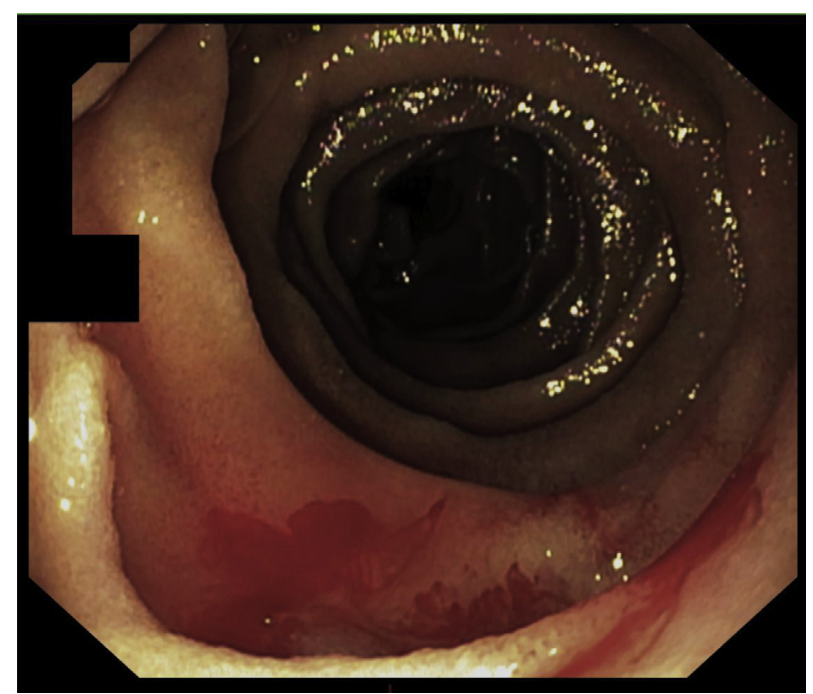

FIGURE 2. Jejunal bleeding as a result of angiodysplasia.

patients with end-stage heart failure; however, increased frequencies of some complications have been observed. The need for long-term anticoagulation to prevent pump thrombosis is a major determinant of the risk of gastrointestinal bleeding, although other mechanisms may also be involved (eg, massive consumption of coagulation factors, von Willebrand disease, and impaired platelet function). The angiodysplasia in particular has been also recognized as a complication after continuous-flow LVAD implantation (narrow pulse pressure associated with nonpulsatile LVADs may lead to hypoperfusion of gastrointestinal vasculature and to vascular dilatation, which increases the formation of arteriovenous malformations). ${ }^{1}$ The incidence of gastrointestinal bleeding ranges across studies from $17.6 \%$ to $40 \%$; the average transfusion requirement is 2 to 4 units of packed red blood cells per bleeding patient. In our case, despite prompt discontinuation of warfarin and treatment of the bleeding source, the patient had recurrent hemorrhage, resulting in an unacceptable number of blood transfusions with associated risks (eg, infections, hemolytic transfusion reaction). Newer generation LVADs, such as the HeartMate II, allow maintenance of a lower target INR, and some authors have even reported the discontinuation of anticoagulation therapy in bleeding patients for months; nevertheless these studies are small and lacking of long-term follow-ups, and, on the other hand, cases of thromboembolism have been reported as well. ${ }^{3}$ LVADs are burdened by potential and observed risks of pump thrombosis, and, as also reported by Guha and colleagues, ${ }^{4}$ no studies have investigated the optimal anticoagulation management in those patients who have gastrointestinal bleeding during LVAD, and they apply in their center their own protocol calling for withholding of all anticoagulation only after the fifth bleeding episode. ${ }^{4}$ 
More recently, Starling and colleagues ${ }^{5}$ reported an unexpected abrupt increase in HeartMate II early thrombosis in a large series population from between March 2011 and January 2013. ${ }^{5}$ Although the exact causes of this phenomenon remain unknown, Starling and colleagues ${ }^{5}$ underline that anticoagulation protocols differ during the last years and among institutions. They therefore speculate that this could be an explanation for early thrombosis.

Apixaban, a direct inhibitor of factor $\mathrm{Xa}$, is a new oral anticoagulant recently introduced into clinical practice. In the Apixaban for Reduction in Stroke and Other Thromboembolic Events in Atrial Fibrillation trial, ${ }^{6}$ apixaban proved to be superior to warfarin in preventing stroke and myocardial infarction and in reducing the risk of intracranial bleeding in patients with atrial fibrillation. Moreover, in a recent comparative analysis of 3 major clinical trials with new oral anticoagulants, Nunes and colleagues $^{7}$ reported that the gastrointestinal bleeding associated with apixaban did not surpass the figures for warfarin. Nevertheless, some aspects of new oral anticoagulants have yet to be clarified. For eg, dabigatran (Phase II Study to Evaluate the Safety and Pharmacokinetics of Oral Dabigatran Etexilate in Patients after Heart Valve Replacement) showed no benefit and an excess risk in patients with mechanical aortic valve replacement when compared with warfarin. ${ }^{8}$ In our multidisciplinary approach, oral anticoagulation with apixaban appeared the only viable alternative to warfarin, in particular because of the lower risk of bleeding associated with this drug.

To the best of our knowledge, this is the first report in the literature that describes the use of anticoagulation therapy with apixaban in a patient receiving LVAD support with recurrent gastrointestinal bleeding and warfarin intolerance. The patient was treated as an outpatient with apixaban for 1 year, and to date there have been no signs of device thrombosis. Our strategy has been dictated by our position between the devil and deep sea. Keeping in mind the absolute need for an anticoagulation strategy, and also in view of the warning of Starling and colleagues, more evidence and longer follow-up are warranted to assess the safety and efficacy of our choice.

\section{References}

1. Harvey L, Holley CT, John R. Gastrointestinal bleed after left ventricular assis device implantation: incidence, management, and prevention. Ann Cardiothorac Surg. 2014;3:475-9.

2. Cuker A, Siegal DM, Crowther MA, Garcia DA. Laboratory measurement of the anticoagulant activity of the non-vitamin K oral anticoagulants. J Am Coll Cardiol. 2014;64:1128-39.

3. Rossi M, Serraino GF, Jiritano F, Renzulli A. What is the optimal anticoagulation in patients with a left ventricular assist device? Interact Cardiovasc Thorac Surg. 2012;15:733-40.

4. Guha A, Eshelbrenner CL, Richards DM, Monsour HP Jr. Gastrointestinal bleeding after continuous-flow left ventricular device implantation: review of pathophysiology and management. Methodist Debakey Cardiovasc J. 2015;11: 24-7.

5. Starling RC, Moazami N, Silvestry SC, Ewald G, Rogers JG, Milano CA, et al Unexpected abrupt increase in left ventricular assist device thrombosis. $N$ Engl J Med. 2014;370:33-40.

6. Hylek EM, Held C, Alexander JH, Lopes RD, De Caterina R, Wojdyla DM, et al Major bleeding in patients with atrial fibrillation receiving apixaban or warfarin: The ARISTOTLE Trial (Apixaban for Reduction in Stroke and Other Thromboembolic Events in Atrial Fibrillation): predictors, characteristics, and clinical outcomes. J Am Coll Cardiol. 2014;63:2141-7.

7. Nunes JP, Rodrigues RP, Gonçalves FR. Comparative analysis and meta-analysis of major clinical trials with oral factor Xa inhibitors versus warfarin in atrial fibrillation. Open Heart. 2014;1:e000080.

8. Eikelboom JW, Connolly SJ, Brueckmann M, Granger CB, Kappetein AP, Mack MJ, et al; RE-ALIGN Investigators. Dabigatran versus warfarin in patients with mechanical heart valves. N Engl J Med. 2013;369:1206-14. 